\title{
EVALUATION OF MEASUREMENT-BASED OPTIMIZATION SCHEMES FOR BATCH DISTILLATION
}

\author{
C. Welz, B. Srinivasan, and D. Bonvin \\ Institut d'Automatique, École Polytechnique Fédérale de Lausanne, \\ CH-1015 Lausanne, Switzerland
}

\begin{abstract}
The standard approach to deal with uncertainty in dynamic optimization is to take a conservative stand. Measurement-based optimization schemes allow reducing this conservatism by using measurements to compensate for the uncertainty. On the example of the productivity optimization of a batch distillation column with a terminal quality constraint, various model-based and model-free optimization schemes are compared. They all use measurements to update the input from batch-to-batch or within a batch. A novel mid-course correction scheme for satisfying the terminal constraint is proposed. Copyright (C)2002 IFAC
\end{abstract}

Keywords: Dynamic optimization, Batch distillation, Batch processes, Batch-to-batch optimization, Run-to-run Optimization, On-line optimization.

\section{INTRODUCTION}

The optimization of batch processes has received increasing attention since it is a natural choice for maximizing productivity. Typically, the quantity of the desired product is maximized at final time while respecting operational path constraints and terminal quality constraints. The classical approach is to apply open-loop input profiles that have been determined off-line. In practical applications, perturbations and uncertainties are present, which may lead to constraint violation or non-optimal operation. To satisfy the constraints despite uncertainty, conservative input trajectories that guarantees feasibility needs to be found (Terwiesch et al., 1994). However, such a conservative strategy is, in most cases, non-optimal.

When suitable measurements are available, they can be used in the optimization scheme to reduce conservatism (Bonvin et al., 2001). Depending on the availability of measurements, the inputs are updated during the batch (intra-batch) or in a batch-to-batch manner (inter-batch). The intra-batch optimization is capable of coping with perturbations that occur during a batch run. The objective of inter-batch optimization is to exploit the repetitive nature of batch processes to find the optimal operating conditions iteratively (Srinivasan et al., 2001).

Two approaches can be distinguished depending on whether or not the model is used at the implementation level (Srinivasan et al., 2002). In the indirect approach, where a model is used for implementation, the estimates of the states and/or parameters are used to compute updated optimal trajectories. In the direct batch-to-batch approach, the parameters of the inputs are updated using a feedback controller (without the use of a model) to meet the terminal constraints. With on-line measurements, the direct approach requires a mid-course correction strategy to satisfy the terminal constraints, for which only very few studies have been reported (Yabuki and MacGregor, 1997). This paper proposes a novel scheme that tracks an off-line determined trajectory.

As an example, the optimal operation of a batch binary distillation column is studied. Numerous 
publications deal with the optimization of batch distillation columns in the case of no uncertainty (Robinson, 1969; Hansen and Jørgensen, 1986; Diwekar et al., 1987; Farhat et al., 1991). The objectrive is to maximize the quantity of distillate at final time, or to minimize the time of operation for a given productivity. Typically, a terminal constraint on the average distillate quality is imposed. In this paper, the problem of maximizing productivity with a terminal constraint on quality will be studied with the reflux ratio being the sole the manipulated variable. Various measurementbased optimization schemes will be evaluated in the presence of uncertainty in the relative volatility and boilup rate, with the measurement of the average distillate composition used to compensate for the uncertainty.

This paper is organised as follows: In the next section, a classification of optimization schemes to deal with uncertainty is undertaken. Section 3 describes the on-line tracking scheme that can be used for tracking terminal constraints. The problem of optimizing a distillation column is presented in Section 4, and various optimization schemes are evaluated on this example in Section 5. Finally, conclusions are drawn in Section 6 .

\section{CLASSIFICATION OF OPTIMIZATION SCHEMES}

The terminal-cost optimization problem with uncertain parameters $\theta$, perturbations $d^{k}(t)$ and measurement noise $v^{k}$ in the $k^{t h}$ batch can be stated mathematically as follows:

$$
\begin{aligned}
\max _{u^{k}(t)} J^{k}= & \phi\left(x^{k}\left(t_{f}\right), \theta\right) \\
\text { s.t. } & \dot{x}^{k}=F\left(x^{k}, u^{k}, \theta\right)+d^{k}(t), x^{k}(0)=x_{0}^{k} \\
& S\left(x^{k}, u^{k}, \theta\right) \leq 0, \quad T\left(x^{k}\left(t_{f}\right), \theta\right) \leq 0 \\
& y^{k}=h\left(x^{k}, \theta\right)+v^{k}(t) \\
\text { given } & z^{j}, \quad \forall j=\{1 \ldots k-1\} \\
\text { or } & y^{k}\left(t_{i}\right), \quad \forall i=\{1 \ldots l\}
\end{aligned}
$$

where $J^{k}$ is the cost function, $u^{k}$ the inputs, $x^{k}$ the states with initial conditions $x_{0}^{k}, S\left(x^{k}, u^{k}, \theta\right)$ the path constraints, and $T\left(x^{k}\left(t_{f}\right), \theta\right)$ the terminal constraints. The measurements at the end of the $\mathrm{j}^{\text {th }}$ batch are represented by $z^{j}, j=\{1 \ldots k-1\}$, and $y^{k}\left(t_{i}\right)$ represents the on-line measurements at time instant $t_{i}$ in batch $k$.

In the presence of uncertainty, the introduction of a security margin (backoff) for active constraints is necessary. Backoffs $\left(b_{S} \geq 0, b_{T} \geq 0\right)$ can added to the path and terminal constraints as in: $S\left(x^{k}, u^{k}, \theta\right)+b_{S} \leq 0, T\left(x^{k}\left(t_{f}\right), \theta\right)+b_{T} \leq 0$. The backoffs are so chosen that the probability of constraint satisfaction is larger than a prespecified confidence level. Since the backoffs affect the optimal solution which in turn affects the backoffs, the backoffs have to be calculated using an iterative scheme (Srinivasan et al., 2002).

Different dynamic optimization schemes are classified in Figure 1 (Bonvin et al., 2001). Possible combinations of the schemes are not considered, whereby certain parameters are adapted on-line and others in a batch-to-batch basis.

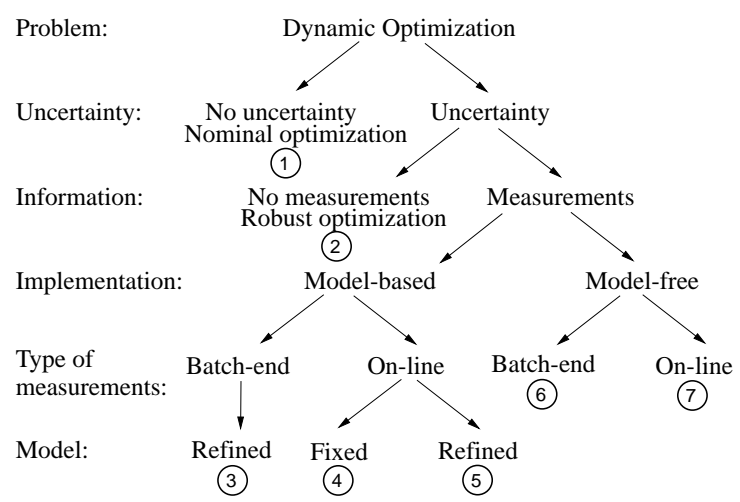

Fig. 1. Classification of dynamic optimization schemes in the presence of uncertainty

\subsection{Nominal optimization}

When uncertainty is neglected, the nominal or expected values of the uncertain parameters are used in the optimization. Thus, the nominal solution may not even be feasible, let alone optimal, in the presence of uncertainty.

\subsection{Robust optimization}

Taking the uncertainty into account explicitly, a robust solution is obtained, for example by considering the worst-case scenario for the constraints and optimizing the cost in an expected sense. Such a conservative solution guarantees feasibility, however the cost is inferior due to the introduction of backoffs.

\subsection{Batch-to-batch optimization, refined model}

When measurements are available at the end of the batch, they can be incorporated into an optimization scheme that updates the input trajectories in a batch-to-batch manner. The optimization scheme consists of two steps:

(i) estimation of the uncertain parameters at the end of each batch, and

(ii) computation of the optimal input trajectories using the refined model.

Both steps use the model explicitly and can become computationally expensive with large models. Additionally, a conflict between identification and optimality objectives may be observed: If, the input it not sufficiently excited, it may not be rich enough to uncover the uncertain parameters. On the other hand, if the input is sufficiently excited, the operation may no longer be optimal. 


\subsection{On-line optimization, fixed model}

When measurements are available during the batch, reoptimization can be executed with the advent of every measurement. It is supposed that reoptimization can be completed in-between the measurements, so that the inputs are updated after each measurement. When a fixed model is used, only the states are estimated and the optimal trajectory is calculated for the remaining of the batch. However, the computed optimal solution can become infeasible, especially towards the end of the batch, due to model inaccuracies.

\subsection{On-line optimization, refined model}

To compensate for the model inaccuracies, the uncertain parameters can also be estimated on-line using the available measurements. The estimated parameters are then used in a refined model for the calculation of the optimal trajectory. When such an approach is used, the issue of persistent excitation need to be addressed explicitly.

\subsection{Batch-to-batch optimization, model-free}

In the model-free implementation schemes, the measurements are used directly to adapt the optimal input. They use the fact that the optimal inputs of (1) consist of various arcs and the inputs can be parameterized as a function of the states and so-called input parameters $\pi, u=$ $\mathcal{U}(x, \pi)$. The input parameterization also includes the switching time between different arcs.

Without loss of generality, assume that all terminal constraints are active. Then, the necessary condition for optimality with parameterization $\pi$ can be stated as follows (Srinivasan et al., 2002):

$$
T=0, \quad \psi=\frac{\partial \phi}{\partial \pi}+\nu^{T} \frac{\partial T}{\partial \pi}=0
$$

where $\nu$ is the vector of Lagrange multipliers for the terminal constraints. The idea of model-free optimization is to satisfy the necessary conditions of optimality (2) despite uncertainty by adjusting the values of $\pi$ using measurements. The necessary conditions consist of two parts: (i) the constraint part $T=0$, and (ii) the sensitivity part $\psi=0$. There is usually considerably more to gain by meeting the constraints than from reducing the sensitivities to zero. Thus, only the satisfaction of terminal constraints will be discussed in this paper.

Consider (i) the batch-end measurements to be the value of terminal constraints, $z^{j}=T\left(x^{j}\left(t_{f}\right)\right)$, (ii) a subset of $\pi, \bar{\pi}$ of dimension $\tau$, which has a large influence on $T$, and (iii) the $\tau \times \tau$ gain matrix, $G: \bar{\pi} \rightarrow z$, between the input parameters $\bar{\pi}$ and the measurements $z$. Then, $G^{-1}$ can be used for decoupling, and the following update law for $\bar{\pi}$ can be used:

$$
\bar{\pi}^{j+1}=\bar{\pi}^{j}+G^{-1} K^{j} z^{j}
$$

This represents an integral control law, where $K^{j}$ is a diagonal gain matrix of dimension $\tau \times \tau$. The other elements of $\pi$ are kept constant.

\subsection{On-line optimization, model-free}

In the previous subsection, a batch-to-batch adaptation methodology that uses batch-end measurements for pushing the system closer to terminal constraints was presented. However, when on-line measurements are available, a mid-course correction methodology to satisfy terminal constraints is necessary. One such scheme will be discussed in the next section.

\section{ON-LINE TRACKING TO MEET TERMINAL CONSTRAINTS}

Constraints in general, and terminal constraints in particular, play an important role in batch optimization problems. As discussed earlier, an operation point quite close to the optimum can be achieved just by meeting the terminal constraints.

With batch-end measurements, only systematic variations, i.e., those that occur the same way in every batch, can be compensated. To handle variations within the batch, it is important to use on-line measurements. So, with on-line measurements, a larger class of uncertainty can be handled, the backoff can be reduced, thereby leading to improved performance. Also, it is wise to use on-line measurements when they are available!

The batch-end measurements that are needed are measurements of the terminal constraints. Since on-line measurements do not directly provide this information, some sort of prediction or extrapolation is needed. Such a prediction is not always robust due to model mismatch and disturbances. Though model mismatch can be handled by refining the model using measurements, the approach suffers typically from lack of persistent excitation.

Suppose the on-line measurements $y\left(t_{i}\right)=T\left(x\left(t_{i}\right)\right)$ are available, i.e., the quantities corresponding to the terminal constraints $T\left(x\left(t_{f}\right)\right)$ are not only measured at the end of the batch but also during the batch. The idea proposed in this paper is to track conservative reference trajectories $y_{r}(t)$ whose main purpose is to guarantee the satisfaction of the terminal constraints at final time, i.e., $y_{r}\left(t_{f}\right)=0$. Then, the adaptation law is given by:

$$
u\left(t_{i}\right)=u_{r}\left(t_{i}\right)+K_{p}\left(y_{r}\left(t_{i}\right)-y\left(t_{i}\right)\right)
$$


where $u_{r}$ and $y_{r}$ are the conservative inputs and output trajectories. The input is constant between sampling instants. Optionally, an integral term can be added to the adaptation law (4). Also, reference trajectories with $y_{r}+b_{T} \leq 0$ can be chosen to provide a safety margin in the presence of measurement noise.

Though this scheme does not use the model for implementation, a model is needed to generate the reference trajectory. If there is no uncertainty (modeling errors and disturbances), then $y\left(t_{i}\right)=$ $y_{r}\left(t_{i}\right)$, and the proposed feedback controller has no effect, $u\left(t_{i}\right)=u_{r}\left(t_{i}\right)$. The role of the feedback controller is therefore to steer the system towards the predicted reference state, thereby rejecting the effect of model uncertainty and disturbances.

It is interesting to note the twist in concept - the model is not adapted to provide a good prediction of the system instead, the input is adjusted so that the system follows the model prediction. Since the model prediction renders the terminal constraint active, following it close enough will push the system towards the terminal constraints.

Note that no optimization nor estimation has to be executed on-line, which makes this method computationally attractive and numerically robust. So, in comparison to the model-based online optimization scheme, the sampling frequency can be higher, the backoff can be reduced, and the cost can be improved.

\section{OPTIMIZATION OF A BATCH BINARY DISTILLATION COLUMN}

\subsection{Modeling}

A batch binary distillation system is considered. The model is based on previous work reported in the literature, see e.g. (Diwekar et al., 1987), (Robinson, 1969), (Farhat et al., 1991) and (Hansen and Jørgensen, 1986). The following assumptions are made: (1) Equimolar overflow, (2) Constant relative volatility, ideal vapor-liquid equilibrium, (3) Equilibrium stages, (4) Negligible vapor holdup, (5) Constant liquid holdup on stages and in condenser, (6) Total condenser, (7) Constant boilup rate.

Considering a column with a total of $p$ equilibrium stages and using the molar balance equation for the holdup in the reboiler and the liquid molar fraction on the various stages and in the condenser, the following model of order $(p+2)$ is obtained:

$$
\begin{aligned}
\dot{M}_{1} & =-f_{d} V \\
\dot{x}_{1} & =\frac{V}{M_{1}}\left(x_{1}-y_{1}+\left(1-f_{d}\right) x_{2}\right)
\end{aligned}
$$

$$
\begin{aligned}
& \dot{x}_{i}=\frac{V}{M_{i}}\left(y_{i-1}-y_{i}+\left(1-f_{d}\right)\left(x_{i+1}-x_{i}\right)\right)(7) \\
& \dot{x}_{c}=\frac{V}{M_{c}}\left(y_{p}-x_{c}\right)
\end{aligned}
$$

$i=2, \ldots, p$, where $x_{i}$ is the molar liquid fraction, $y_{i}$ the molar vapor fraction and $M_{i}$ the holdup on Stage $i$. Stage 1 refers to the reboiler and Stage $p$ to the top of the column. The model uses the property that the composition of the liquid flow entering the top stage corresponds to the composition in the condenser, $x_{c}$, i.e., $x_{p+1}=x_{c}$. $M_{c}$ is the holdup in the condenser. The ratio $f_{d}$ of the distillate to boilup rate, $f_{d}=\frac{D}{V}$, is considered as the manipulated variable. The vapor-liquid equilibrium relationship is:

$$
y_{i}=\frac{\alpha x_{i}}{1+(\alpha-1) x_{i}}, \quad i=1, \cdots, p
$$

where $\alpha$ is the relative volatility. The model parameters and the initial conditions are given in Table 1. The composition of the accumulated distillate, $x_{d}$, is assumed to be measured with the sampling time, $t_{s}$ is given by:

$$
x_{d}(t)=\frac{\sum_{i=1}^{p} x_{i}(t) M_{i}(t)-x_{i}(0) M_{i}(0)}{M_{1}(t)-M_{1}(0)}
$$

Table 1. Model Parameters and initial conditions, $i=2, \cdots, p$

\begin{tabular}{ccc|ccc}
\hline$p$ & 10 & & $V$ & 15 & $\mathrm{kmol} / \mathrm{h}$ \\
$t_{f}$ & 10 & $\mathrm{~h}$ & $x_{d, \text { des }}$ & 0.9 & $\mathrm{kmol} / \mathrm{kmol}$ \\
$t_{s}$ & 30 & $\min$ & $M_{1}(0)$ & 100 & $\mathrm{kmol}$ \\
$\alpha$ & 1.5 & & $x_{1}(0)$ & 0.5 & $\mathrm{kmol} / \mathrm{kmol}$ \\
$M_{i}$ & 0.2 & $\mathrm{kmol}$ & $x_{i}(0)$ & 0.5 & $\mathrm{kmol} / \mathrm{kmol}$ \\
$M_{c}$ & 2 & $\mathrm{kmol}$ & $x_{c}(0)$ & 0.5 & $\mathrm{kmol} / \mathrm{kmol}$ \\
\hline
\end{tabular}

The objective is to maximize the quantity of accumulated distillate for a given batch time $t_{f}$ with a terminal constraint on $x_{d}\left(t_{f}\right)$. Additionally, there are path constraint on the manipulated input $f_{d}$. The optimization problem is mathematically stated as follows:

$$
\begin{aligned}
\max _{f_{d}(t)} J= & M_{1}\left(t_{0}\right)-M_{1}\left(t_{f}\right) \\
\text { s.t. } & \text { Diff. Alg. Equations }(5)-(10) \\
& 0 \leq f_{d}(t) \leq 1 \\
& x_{d}\left(t_{f}\right) \geq x_{d, \text { des }}
\end{aligned}
$$

\subsection{Characterization of the optimal solution}

The optimal solution obtained numerically consists of three intervals:

(1) Full reflux $\left(f_{d}=0\right)$,

(2) A nearly linear arc to represent the compromise between quality and productivity,

(3) No reflux $\left(f_{d}=1\right)$. 
As a result, the input can be parameterized using the following four parameters: the two switching times $t_{1}$ and $t_{2}$ and the parameters for the linear profile, the level $l$ and the slope $s$. The parameterized optimal input trajectory is illustrated in Figure 2. This parameterization results in the optimal cost $J=22.73 \mathrm{kmol}$, and the input parameters are $\pi=\left[\begin{array}{llll}t_{1} & t_{2} & l & s\end{array}\right]^{T}=\left[\begin{array}{lllll}1.02 & 9.88 & 0.1748 & -0.0039\end{array}\right]^{T}$.

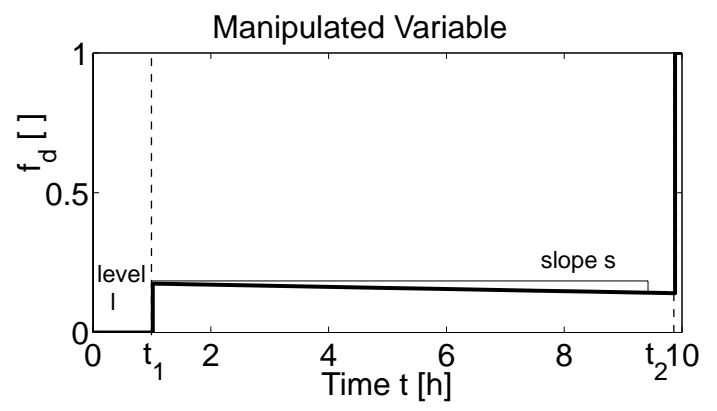

Fig. 2. Parameterization of the input $f_{d}$.

\section{EVALUATION OF VARIOUS SCHEMES}

In order to provide a realistic test scenario, the following uncertainty is considered:

- Parametric uncertainty: Fixed but unknown relative volatility in the range $\alpha=\left[\begin{array}{ll}1.4 & 1.6\end{array}\right]$

- Perturbation: Boilup rate equally distributed in the range $V=\left[\begin{array}{ll}13 & 17\end{array}\right] \mathrm{kmol} / \mathrm{h}$, changed every $2.5 h$

- Measurement noise: Product composition $x_{d}$ with $5 \%$ multiplicative gaussian noise

The value $\alpha=1.5$ is used all simulations. However, this value is not disclosed to the various optimization schemes that start with the worstcase value $\alpha=1.4$. The costs reported in Table 2 are calculated on the basis of 50 realizations with respect to perturbation and measurement noise. The backoffs are introduced so that in every case the constraint satisfaction is $99 \%$.

The results of the various optimization schemes are compared in Table 2. The loss in performance is calculated as: Loss $=\left(J_{\text {nom }}-J\right) / J_{\text {nom }}$, where $J$ is the actual cost and $J_{\text {nom }}$ the nominal cost without parametric uncertainty, perturbation and measurement noise. The optimal profiles could be updated either (i) on-line, (ii) batch-to-batch, or (iii) both. The third case is not considered here. Thus, with on-line schemes, the improvement shown in Table 2 is that obtained from a single batch.

(1) Nominal case: When the nominal input trajectory is applied open-loop in the presence of uncertainty, the terminal constraint is satisfied in only $53 \%$ of the realizations.

(2) Robust case: If measurements are not available, constraint satisfaction is guaranteed by using
Table 2. Comparison of cost and required backoff. The numbering refers to subsections of Section 2

\begin{tabular}{|c|c|c|c|c|}
\hline & Method & $\begin{array}{c}\text { Cost } \\
J[k m o l]\end{array}$ & $\begin{array}{c}\text { Backoff } \\
b_{T}\end{array}$ & $\begin{array}{c}\text { Loss } \\
{[\%]}\end{array}$ \\
\hline 1 & $\begin{array}{l}\text { Open loop, } \\
\text { nominal input }\end{array}$ & 22.73 & infeasible & 0 \\
\hline 2 & $\begin{array}{l}\text { Open loop, } \\
\text { robust input }\end{array}$ & 14.98 & 0.042 & 34.1 \\
\hline 3 & $\begin{array}{l}\text { Batch-to-batch, } \\
\text { refined model }\end{array}$ & 20.32 & 0.015 & 10.6 \\
\hline 4 & $\begin{array}{l}\text { On-line, } \\
\text { fixed model }\end{array}$ & 20.20 & 0.015 & 11.1 \\
\hline 5 & $\begin{array}{l}\text { On-line, } \\
\text { refined model }\end{array}$ & 20.41 & 0.013 & 10.2 \\
\hline 6 & $\begin{array}{l}\text { Batch-to-batch, } \\
\text { model-free }\end{array}$ & 20.83 & 0.012 & 8.4 \\
\hline 7 & $\begin{array}{l}\text { On-line, } \\
\text { model-free }\end{array}$ & 20.64 & 0.010 & 9.2 \\
\hline
\end{tabular}

the worst-case parameters in the optimization, $\alpha=1.4$ and $V=17 \mathrm{kmol} / \mathrm{h}$.

(3) Batch-to-batch, refined model: In the batchto-batch optimization scheme, the uncertain parameters are estimated by least-squares estimation using the batch-end measurements of average distillate composition. The refined model is then used to update the input parameters. The optimal cost is reached in about 5 batches (Figure 3), but the cost changes significantly from batch to batch due to the perturbation and measurement noise.

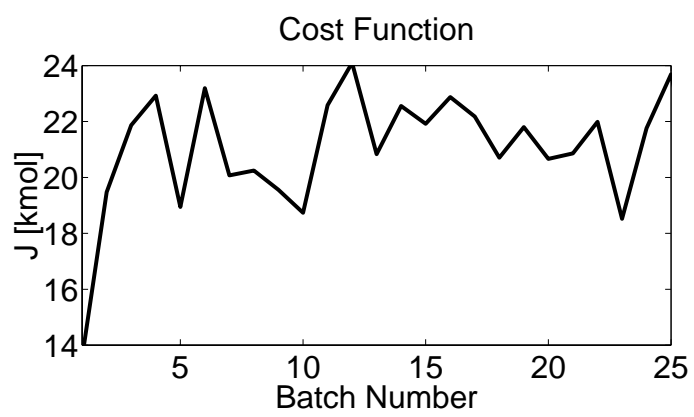

Fig. 3. Evolution of the cost function for the batch-to-batch, refined model scheme.

(5) On-line case, fixed model: If the measurement of $x_{d}$ is available on-line, the current state of the system can be estimated and used for reoptimization. However, such a procedure is slightly inferior to batch-to-batch schemes due to wrong parameters. Also, the computed optimal input becomes infeasible towards the end of the batch and some heuristic approaches need to be used to circumvent the problem.

(4) On-line, refined model: Here, the uncertain parameters are also estimated. The forgetting factor $\lambda=0.94$ is used in the least-squares estimation method. It was seen that the parameter estimates do not actually coincide with the true values. This can be attributed to, bias in estimation, a lack of sufficiently exciting signals, and infrequent measurements. Also, since the measurements are 
only available after the switching time $t_{1}$, this input parameter cannot be adapted in any on-line optimization method.

(6) Batch-to-batch, model-free: Among the input parameters $\pi=\left[t_{1}, t_{2}, l, s\right]$, the switching time $t_{2}$ does not change with the uncertainty considered and need not be adapted. The parameter with the strongest influence on the terminal quality constraint is the level $l$. So, a simple integral control law as in (3): $l^{j+1}=l^{j}+G^{-1} K^{j} T^{j}$, with $G^{-1}=1.5$, is used for batch-to-batch optimization. In addition, the controller gain is reduced with the batch number $j: K^{j}=j^{-0.9}$. With such a scheme, the optimal cost is reached is about 5 batches (see Figure 4). Note that the variations in cost are less than with the batch-to-batch, refinedmodel scheme (Figure 3).

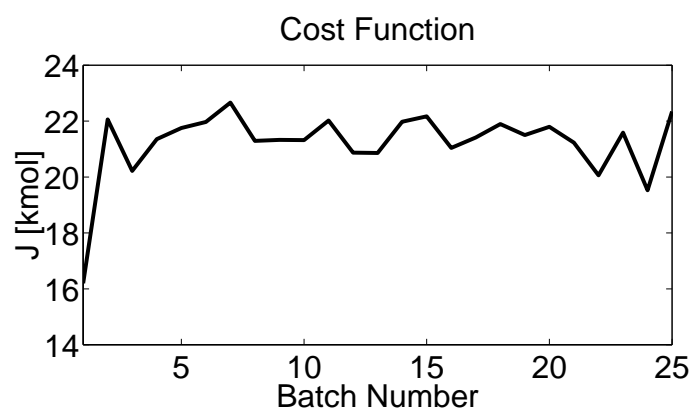

Fig. 4. Evolution of the cost for the model-free batch-to-batch scheme.

(7) On-line case, model-free: Assuming that $y\left(t_{i}\right)=$ $x_{d}\left(t_{i}\right)$ can be measured without any delay, a trajectory $x_{d_{r}}(t)$ is tracked. In this case, the conservative trajectory computed off-line using robust optimization techniques was considered as $x_{d_{r}}(t)$. Here, trajectory tracking closely resembles the constant distillate purity method of operation. The backoff $b_{T}=0.01$ is added to the reference trajectory and tracking is done using a PI-controller with $k=1.2, T_{i}=1.6$. Figure 5 shows that though the reference trajectory is not perfectly tracked, the terminal constraint is attained at the end of the batch.

The performance can be improved in two different ways: (i) If the measurements are available more frequently, it is possible to increase the sampling time, since on-line computation is minimal. With the sampling time $t_{s}=3 \mathrm{~min}$, the backoff can be reduced to $b_{T}=0.009$ and the performance is only $7 \%$ inferior to the nominal solution. (ii) Performance improvement can be achieved with a combination of on-line tracking and batch-to-batch adaptation of the reference trajectory $x_{d_{r}}(t)$.

\section{CONCLUSION}

Several optimization schemes that use measurements to reduce conservatism (necessary in the presence of uncertainty) have been presented. The

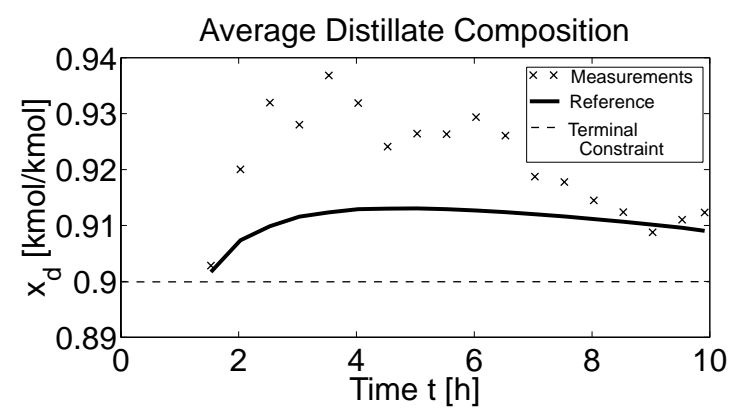

Fig. 5. Reference trajectory and measurements for the on-line model-free scheme.

methods were applied to a simulated batch binary distillation with terminal cost and path and terminal constraints. A novel scheme was proposed to track a reference trajectory on-line, the purpose of which is to bring the system to the terminal constraints. This method is numerically robust since no parameter estimation nor trajectory reoptimization is required on-line. Future work will investigate the application of the method to multiinput systems with several terminal constraints.

\section{REFERENCES}

Bonvin, D., B. Srinivasan and D. Ruppen (2001). Dynamic optimization in the batch chemical industry. In: Chemical Process Control-6. Tuscon, AZ.

Diwekar, U. M., R. K. Malik and K. P. Madhavan (1987). Optimal reflux rate policy determination for multicomponent batch distillation columns. Comp.Chem.Eng. 11(6), 629-637.

Farhat, S., L. Pibouleau, S. Domenech and M. Czernicki (1991). Optimal control of batch distillation via nonlinear programming. Chem.Eng.Process. 29, 33-38.

Hansen, T. H. and S. B. Jørgensen (1986). Optimal control of binary batch distillation in tray or packed columns. Chem.Eng.J. 33, 151155.

Robinson, E. R. (1969). The optimization of batch distillation operations. Chem.Eng.Science 24, 1661-1668.

Srinivasan, B., C. J Primus, D. Bonvin and N. L. Ricker (2001). Run-to-run optimization via generalized constraint control. Control Eng. Practice 9, 911-919.

Srinivasan, B., E. Visser, D. Bonvin and S. Palanki (2002). Optimization of batch processes: II. Handling uncertainty using measurements. Comp.Chem.Eng.

Terwiesch, P., M. Agarwal and D. W. T. Rippin (1994). Batch unit optimization with imperfect modeling - A survey. J.Proces.Contr. 4, 238-258.

Yabuki, Y. and J. F. MacGregor (1997). Product quality control in semi-batch reactors using mid-course correction policies. In: IFAC ADCHEM'97. Banff, Canada. pp. 189-194. 